\title{
2018 Proceedings
}

\author{
Afterlife \\ Sanjay Guria, Yoojin Chung, and Susan Ashdown, Cornell University, USA
}

Keywords: textiles, recycling, upcycling, sustainability

Context, Purpose and Background: In today's throwaway culture, contemporary designers are contributing to the growing movement of sustainable and ethical fashion through innovative means by reassessing the concept of value. As Kate Fletcher notes in her manifesto on "Clothes That Connect", "We will see beauty and greatness in garments that value process, participation and social integration, in pieces that advance relationship between people and the environment." (Fletcher, 2007, p. 123). To achieve this, some designers are employing modern technology, while others are stimulated by traditional repositories of knowledge.

AfterLife is inspired by the indigenous wisdom of upcycling and frugality embedded in the utilitarian textiles and clothing of Japan, specifically the boro textiles and the kimono. Traditional kimonos were designed to loom width specifications with no wastage and were usually sewn by hand with exceptional skill. They were and are practical and versatile, as the size-free feature allows for easy adaptation of weight and height. Furthermore, layering and different material usage allows it to be practical for all seasons. In contrast, the 'boro' are textile heirlooms that have been used, broken, worn to tatters and those that are extensively repaired and patched (Wada, 2014). AfterLife is a harmonious amalgamation of these two inspirations while continuing to maintain their individuality.

Our aim was to create a sophisticated ensemble that will appeal to a western audience while rejoicing its eastern influence. Though our starting point had the common thread of thoughtful use of materials and personalization, we translated it differently through the design of individual components of the ensemble. Both the boro and kimono are an assemblage of unique hand-sewn shapes and embedded stories. Emulating this, the double layered outer garment in a single color, lightweight organza fabric denotes the cover of a story book, through which the inner pure silk patchwork sheath is visible where each textile piece assembled represents a unique story that is hand-stitched together to form an anthology of collective lived experiences.

Process: Once the concept was finalized with the aid of rough sketches, as a team we pooled our resources to flesh out the details. The fabric for the ensemble was consciously sourced from a local reuse store. Since it was a post-consumer waste fabric, we had a limited colour palette and fabric quantity per colour to play with. The patchwork for the inner sheath was carefully detailed on an actual size stencil, ensuring not only that the colours were visually balanced, but also that the fabrics were judiciously cut from the waste pieces. All the pieces for the patchwork were then pieced one-at-a-time using kantha, an ancient form of hand-stitch embroidery from India. Fusing was used to reduce the fraying of the patches, so as to maintain the premium feel we were targeting to achieve. True to its inspiration, the pattern pieces for the kimono are laid and cut in a

Page 1 of 3

@ 2018, International Textile and Apparel Association, Inc. ALL RIGHTS RESERVED ITAA Proceedings, \#75 - http://itaaonline.org 
manner that has zero waste. A striking feature of the kimono jacket is the innovative use of additional apparel compositions. The outer coat has twice the number of sleeves and collars than a regular coat emphasizing the fabric saved by making the patterns in a rectangular shape.

Contribution: AfterLife contributes to knowledge about how design choices can have sustainable impact on the environment and people. The process of fabric construction used not only adds uniqueness to the ensemble but also reduces machine sewing, thereby saving on the energy cost. While the use of darts and style lines helps to shape the garment to the human curves, it also helps in considered use of the small fabric pieces to create the different garment parts. The facing of the kimono incorporated any textile waste during construction for aesthetics. It shows respect for traditional knowledge streams and skills and invites the audience to delve deeper into our own thought processes. AfterLife is a wearable conceptual piece for women created by re-using material that would have otherwise ended up in a landfill. In the process, we have seen value in what would be considered waste conventionally and converted it into a precious one-of-a-kind heirloom worth living many lives.

\section{References:}

Dalby, L. (2001). Kimono: Fashioning culture. Seattle: University of Washington Press.

Fletcher, K. (2007). Clothes that Connect. In Chapman, J. \& Grant, N. (Eds.), Designers, Visionaries and Other Stories: A Collection of Sustainable Design Essays (pp. 118-32). London and Sterling, VA: Earthscan.

Wada, Y. (2014). Boro Mottainai. In Aronowsky, C.A. (Ed.), On slowness (pp. 41-48). Paris: Vestoj. 

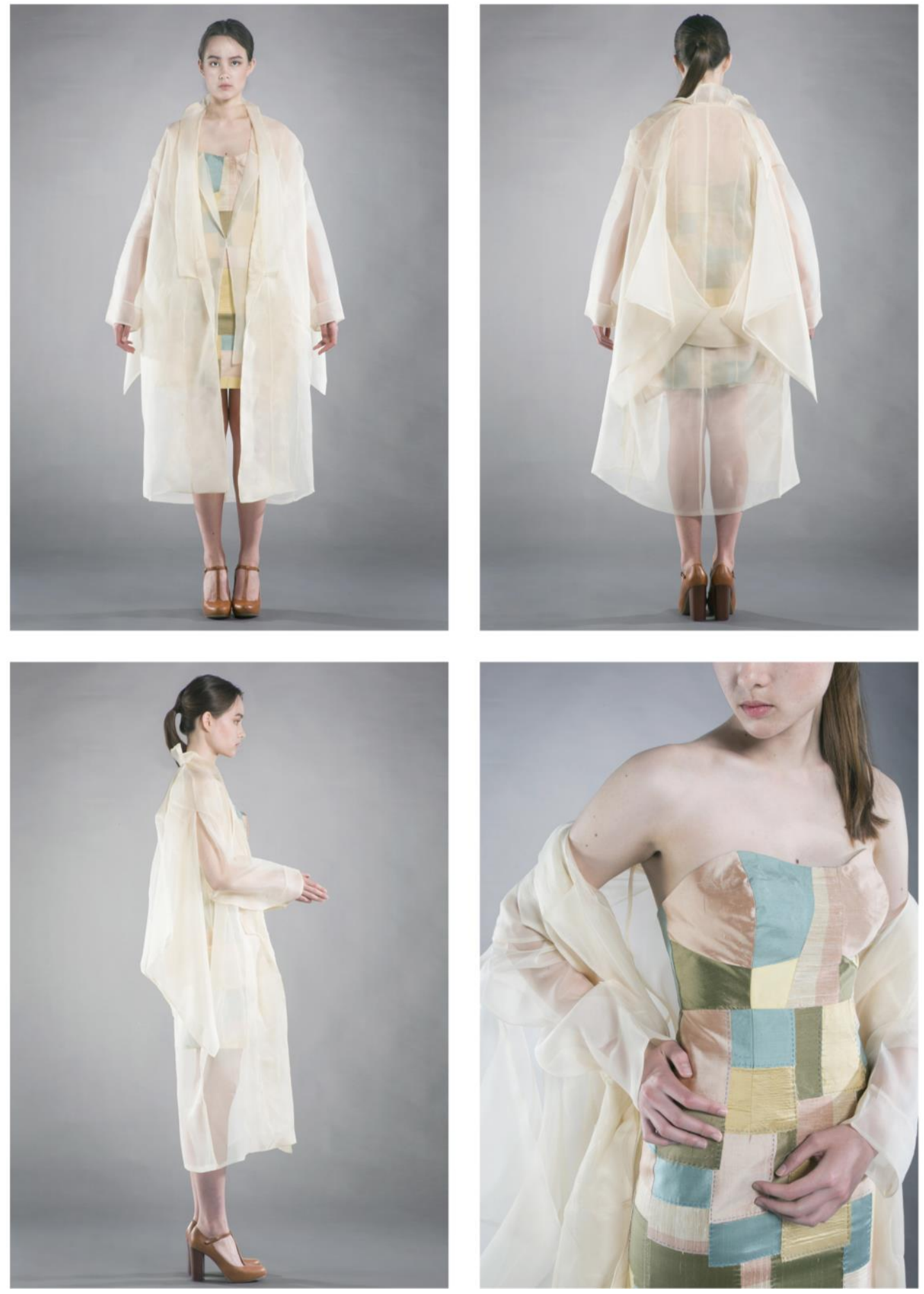

Page 3 of 3

() 2018, International Textile and Apparel Association, Inc. ALL RIGHTS RESERVED

ITAA Proceedings, \#75 - http://itaaonline.org 